\title{
The investigation of the impact of basic operational parameters on the dynamics of water jacket in a biomass boiler using numerical and experimental methods
}

\author{
Mateusz Szubel ${ }^{1}$, Mariusz Filipowicz ${ }^{1}$ \\ ${ }^{1,}$ a AGH University of Science and Technology, Faculty of Energy and Fuels, 30 Mickiewicza Av.30-059 Krakow, Poland
}

\begin{abstract}
Biomass boiler application, despite its many advantages, is dependent upon many technical aspects, which require tests and optimization. Because of practical constraints, one of the most problematic areas of research is the analysis of phenomena occurring inside the water jacket of the boiler during the combustion process. The issue referred to above is significant due to its direct impact on the heating up of the operating medium for current power of the device and the total efficiency. The paper presents the analytical possibilities of the operating medium in a biomass boiler water jacket. The experimental works conducted as a part of the study were performed using an actual device - EKOPAL RM 40 straw boiler. They were aimed at defining the values of significant boundary conditions. Resistance thermometers and K-type thermocouples connected to a data acquisition system were placed in selected points of the water jacket and the combustion chambers to allow the monitoring of the conditions of the water heating process during biomass combustion. A measurement of inlet water mass flow rate was performed. To develop a numerical model of heat transfer into the water jacket, ANSYS CFX software was applied. The results of the experiments and simulations were compared and discussed. The paper describes the methodology and instruments used to perform the experimental studies, as well as some optimization solutions developed based on the results of the numerical analysis.
\end{abstract}

\section{Introduction}

In the preindustrial era, biomass was the basic carrier of primary energy. Currently, the importance of biomass is growing, mainly due to the greenhouse effect and seeking diversification of the energy sources. Presently, lignin-cellulose type biomass is of the highest significance to the energy sector. Combustion, cocombustion and gasification need to be mentioned as three methods of biomass use for energy production [1].

Biomass for energy production can be obtained from side-products of current agriculture and forestry, from forest logging and from dedicated plantations. Forest logging for biomass, of course, is likely to be limited for environmental reasons. On the other hand, if biomass is grown sustainably, its production and use creates no net build-up of carbon dioxide (CO2) in the atmosphere, because the total $\mathrm{CO} 2$ which is released in the combustion processes is offset by $\mathrm{CO} 2$ consumed in photosynthetic processes [2].

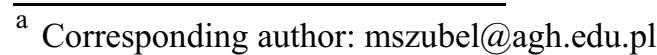

\section{Combustion technologies based on biomass}

In the preindustrial era, biomass was the basic carrier of primary energy. Currently, the importance of biomass is growing, mainly due to the greenhouse effect and seeking diversification of the energy sources. Presently, lignin-cellulose type biomass is of the highest significance to the energy sector [3].

From the energy generation point of view, wastes which are completely useless in agriculture are the most valuable form of biomass. Because of that, it is reasonable to develop energy technologies based on biofuels such as rape straw or sunflower straw.

Currently, the most common energy technologies using biomass are based on direct combustion.

In the case of rural areas it is always worthwhile to analyse the biomass potential from the heat production point of view. Cost effective heating using own straw 
is possible not only in case of households, but also livestock buildings and greenhouses [3].

Low bulk density $\left(40-60 \mathrm{~kg} / \mathrm{m}^{3}\right)$ is a fundamental drawback of straw. Such a fuel requires compaction before transportation. Usually the process is carried out on the field. The results of the compaction are cylindrical or cubic bales. The form of the bales is important from the point of view of the structure of boilers. Straw can be burned in form of bales, chaff (chopped straw), bricket or granules.

In small heating units (up to $1 \mathrm{MWt}$ ), baled straw can be burned on a fixed flat grate with periodic fuel loading or, after crushing, on a fixed sloping grate. The control of the combustion processes is carried out using a fan connected to a digital controller implementing an algorithm, usually based on the measurement of temperature and the content of oxygen at the exhaust [3].

\section{Characteristics of the analysed heating unit}

The study described in the paper was devoted especially to the investigation of the water jacket dynamics based on the example of EKOPAL RM40 $180 \mathrm{~kW}$ biomass boiler. The heating device is an integral part of the experimental installation located in the AGH University of Science and Technology, in the Faculty of Energy and Fuels. The analyzed boiler is designed for periodical straw combustion. Major elements of the commercial version of the unit are marked on Figure 1.

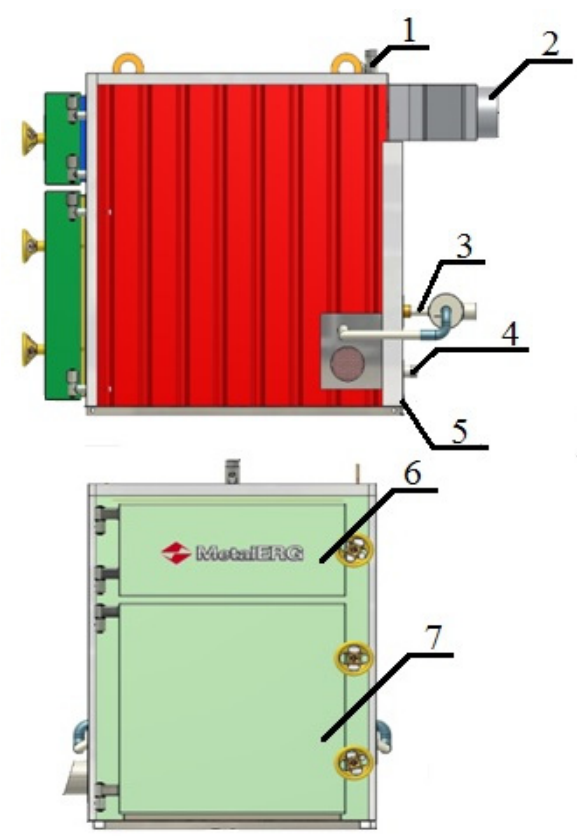

Figure 1. Major elements of the EKOPAL RM40 boiler : 1. Output connector, 2. Combustion flue, 3. Air collector, 4. Input connector, 5. Blowdown, 6. Cleanout for heat exchanger, 7. Door of the primary combustion chamber. Based on [4].

The device operates in a so-called two stage air supply system relying on a manifold installed on the back wall. The primary as well as the secondary air is supplied using the same blowing fan. Seven parallel air ducts are responsible for providing the air for the gasification, which proceeds in the primary combustion chamber. Two extra pipes are connected through the side walls of the secondary combustion chamber, providing secondary air directly to the secondary combustion chamber.

The fan provides primary and secondary combustion air. In the bottom part of the primary chamber, fuel combustion takes place in controlled oxygen deficiency conditions. The originated gases flow into the secondary chamber, lined with chamotte, and mix up with secondary air. In this chamber, the post-combustion gases are generated. From the afterburner, gases flow into the heat exchanger. This is the so called "counter-combustion system", which assures the correct process of straw gasification and combustion of generated syngas. As a result, low levels of carbon monoxide (CO) in the exhaust are achieved [4].

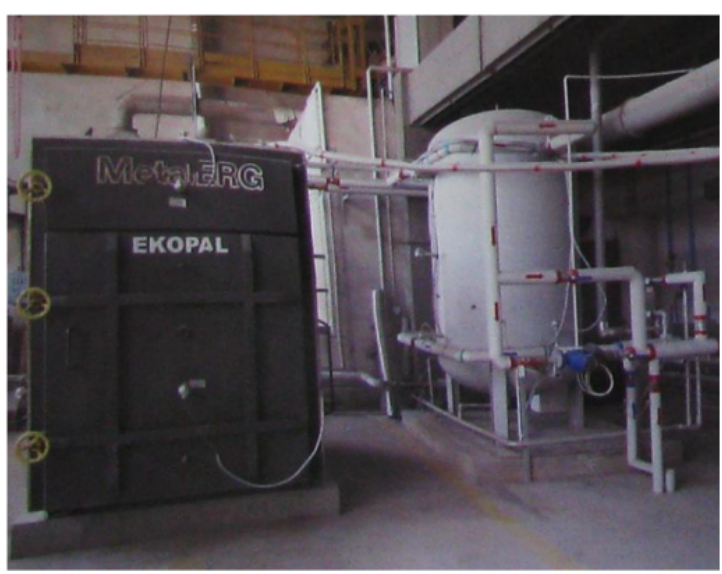

Figure 2. View of the experimental EKOPAL RM40 biomass boiler system, located in the Faculty of Energy and Fuels of the AGH University of Science and Technology in Krakow.

Such type of boilers operates only in an open hydraulic system. The boilers are fitted to the heating installation via a storage tank. The system of the heating unit connected to the buffer tank has been presented in Figure 2. The operating medium, which flows through the water jacket and between boiler and the buffer tank is forced by the pump equipped with an inverter controlled by PLC. The maximum volume flow rate is $8.5 \mathrm{~m}^{3}$ per hour. The water volume of the primary loop (water jacket, buffer tank and pipes) is about $5.5 \mathrm{~m}^{3}$. A storage tank of a proper size is very important to the efficient operation of the heating system. The unit burns fuel efficiently - with levels of energy conversion efficiency higher than $83 \%$ in case of nominal operation conditions. In the commercial version of the device, the burning rate is controlled by a micro-processor controller which always maintains optimal conditions in the combustion chamber. In laboratory conditions, the control system of the process parameters is realized using PLC, which allows for simultaneous observation and data collection. It was possible to provide the monitoring of many measurement points related to different types of physical and chemical process parameters (such as temperature, 
exhaust composition, air and operating medium mass flow as well as many others).

\section{Experimental study of the EKOPAL RM 40 biomass boiler}

Examples of two measurement results, significant from the point of view of the analyses, have been presented in Figures $4-7$. Both tests were performed in identical conditions (fuel load, air supply, operating medium flow etc.), to investigate the repeatability of phenomena occurring in the system. Figures 4 and 5 present the distribution of temperature in chosen points of the combustion chamber during the combustion process.

The labels of curves exhibited in figures 4-5 refer to the following measurement points (Figure 3): DR thermocouple situated in the direct vicinity of the main door of the combustion chamber (at about 2/3 of the height); RG - floor, next to the right wall of the primary combustion chamber; LE - central point of the left wall; SC - top part of the secondary combustion chamber, HE - sensor in the heat exchanger, about $750 \mathrm{~mm}$ from the inlet of the exchanger.

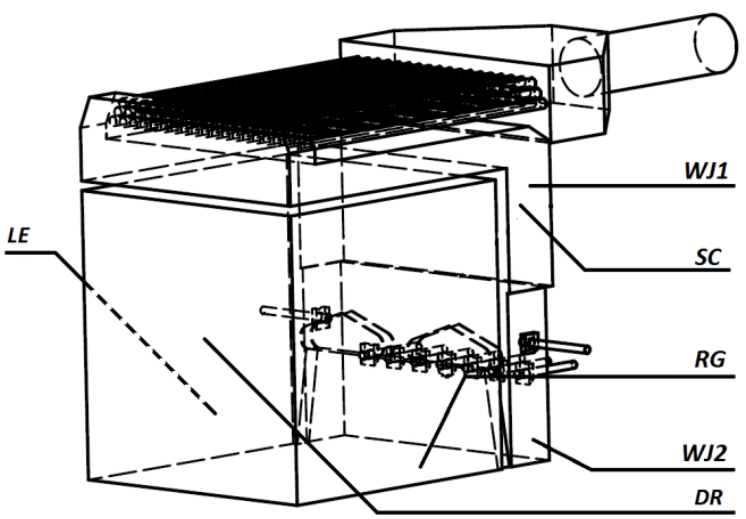

Figure 3. Location of the measurement points.

Each of the sensors, except HE, was placed at a distance of about $150 \sim 160 \mathrm{~mm}$ from the wall on which they were installed.

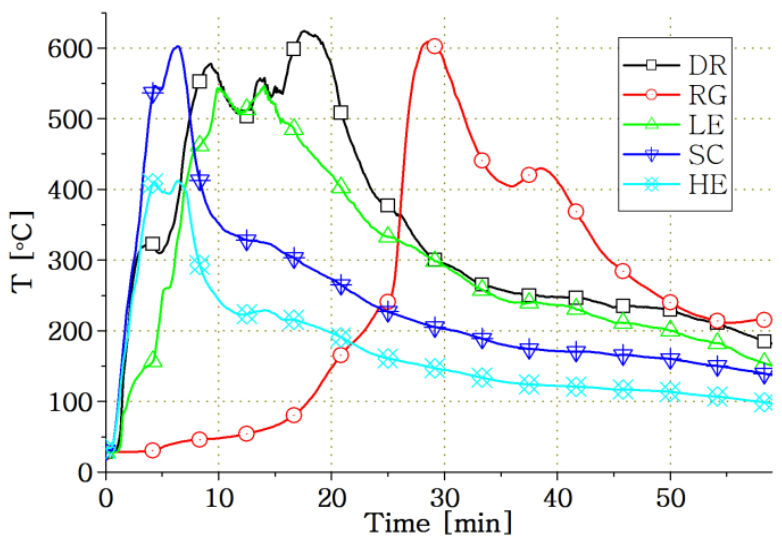

Figure 4. Experiment results of the temperature distribution in selected points of the combustion chambers - first analysis.
In the first stage of the combustion process, the curves in Figures 4 and 5 were similar, which allows to assume some conditions and parameters for further theoretical analyses. The significant difference between the temperature amplitude and the shape of the RG curve is a result of the fact that ignition of fuel proceeds with the use of a dedicated hole in the left rear bottom corner of the primary chamber and the process of flame propagation is transient and random.

Figures 6 and 7 present the process of the heating of the operating medium inside the water jacket. the IN and OUT symbols denote the inlet and outlet temperature, while $\mathrm{WJ} 1$ and $\mathrm{WJ} 2$ describe the temperature in the rear bottom and top corners of the water jacket (Fig. 3).

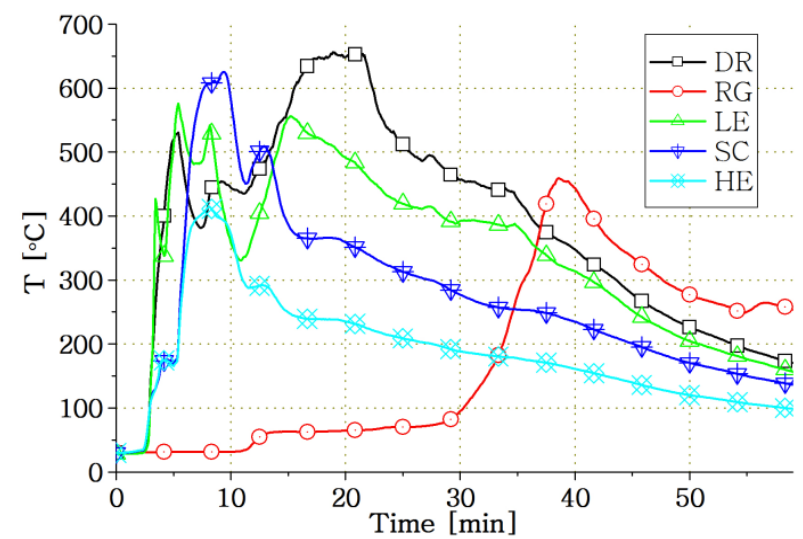

Figure 5. Experiment results of the temperature distribution in selected measurement points of the combustion chambers second analysis.

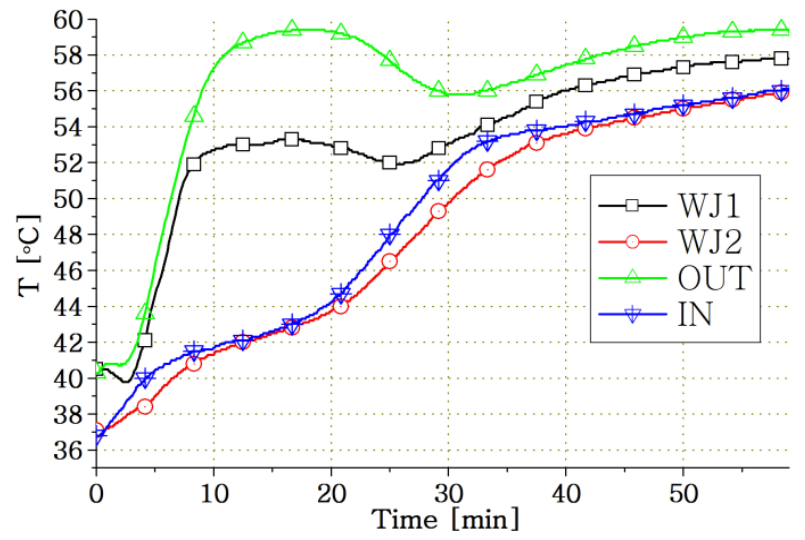

Figure 6. Experiment results of the temperature distribution of the operating medium in selected measurement points of the water jacket - first analysis.

It is evident that the process of medium heating is similar in both cases - small differences are related to the initial conditions of the experiment (initial temperature of water in the buffer tank influences the inlet temperature and the conditions in the bottom region of the water jacket). 


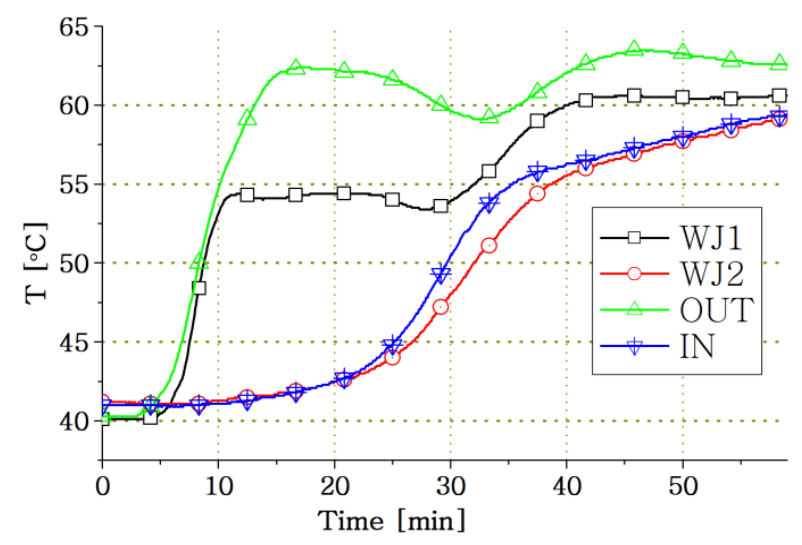

Figure 7. Experiment results of the temperature distribution of the operating medium in selected measurement points of the water jacket - second analysis.

\section{CFD analysis in a study of small scale heating units}

The experimental works devoted to the determination of parameters of the heating units require a lot of effort, especially due to the necessity of providing the fuel, the fact that the process is time-consuming, the costs of staff and installation, safety and many other aspects. Moreover, in case of the optimization process it is necessary to make design changes in existent devices or construct a series of prototypes.

Even a well-equipped experimental system, such as the one referred to above allow only achieving results for points in which sensors are situated. To gain more detailed information about the studied case it is recommended to implement numerical tools such as Computational Fluid Dynamics, which provides the possibility to analyse phenomena in all areas of the devices, even if the studied region is out of stock for the needs of experimental measurement.

\section{Numerical analysis of the operating medium in the water jacket of the EKOPAL RM40 boiler}

To perform a numerical analysis of the biomass boiler water jacket operation, the ANSYS CFX commercial code has been used. The spatial geometry has been designed using Autodesk Inventor 2015 software. The project prepared in this way was transferred to DesignModeler in ANSYS WORKBENCH, where adaptation of the geometry to the process of meshing has been performed. While preparing the processing in ANSYS Meshing, sectioning of the geometry blocks to appropriate shape (for the purpose of generation of good quality grid) and size was performed.

The hex dominant and tetrahedrons method of meshing has been implemented for various assumed regions of the whole geometry. This allowed to optimize the number of computational elements, thus decreasing the time of computation and increasing the quality of the grid. Tetrahedrons were used in more complicated regions, such as the vicinity of the water distribution ducts and untypical shape elements. As a result of the meshing process, 1052030 elements were created.

Because of the relatively small gradient of the temperature between the interior of the water jacket and external surface of the device, it was assumed that there is no heat exchange between the areas mentioned above, and consequently there are no losses caused by thermal insulation of the boiler body (except for losses caused by the connectors between the door of the chamber and the body, which were not considered).

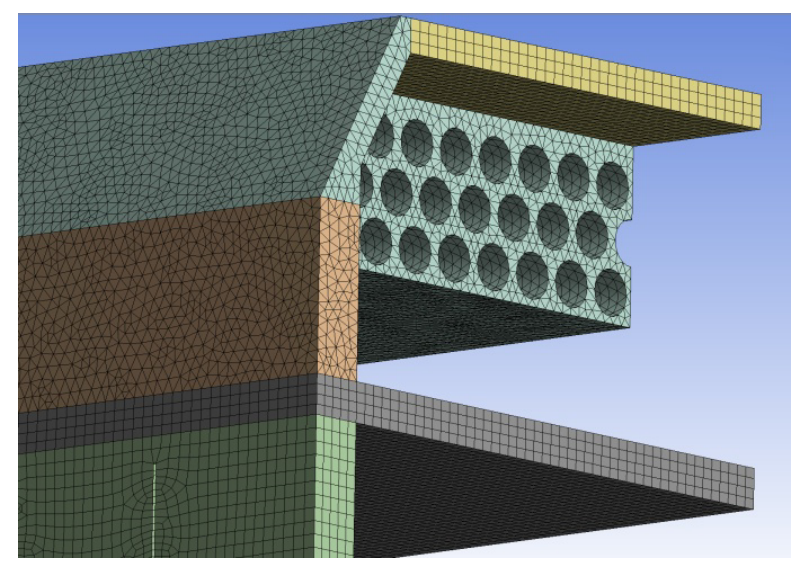

Figure 8. Details of the computational grid generated for the spatial geometry.

Details of the computational grid have been presented in figure 8 . The difference between neighbouring areas is visible in case of side walls (hexagonal elements) and bottom/top wall (tetrahedrons). Figure 9 presents a general view of the spatial geometry after processing in the ANSYS Meshing software. The numbers indicate the areas of the device: 1 - primary combustion chamber, 2 - secondary combustion chamber (vertical section), 3 - dust separator, 4 - inlet to the heat exchanger, 5 - inlet of the water domain, 6 - outlet from the water domain.

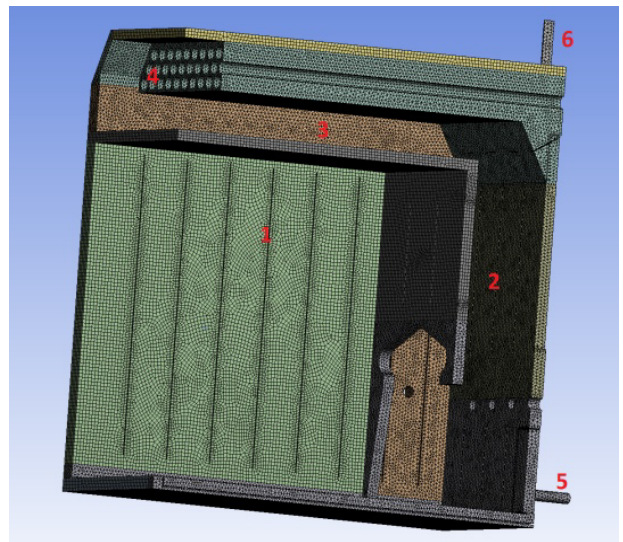

Figure 9. General view of the water jacket geometry.

The geometry, along with the imposed computational grid, has been transferred to the pre-processor. The ANSYS CFX has been used to prepare domain characteristics and boundary conditions. 
The basic mathematical equations $(1-3)$ listed below have been implemented to the model:

continuity equation:

$$
\frac{\partial \rho}{\partial t}+\nabla(\rho v)=0
$$

momentum equation:

$$
\frac{(\partial \rho v)}{\partial t}+\nabla \cdot\left(\rho v_{i} v_{j} v_{k}\right)=\rho g+\nabla \cdot \Gamma,
$$

energy equation:

$$
\frac{(\partial \rho u)}{\partial t}+\nabla \cdot(\rho u v)=\nabla(\lambda \nabla T)+(\Gamma \nabla v),(3)
$$

where $\rho$ density, $\mathrm{kg} \mathrm{m}^{-3} ; t$ time, $\mathrm{s} ; v$ velocity tensor characterised by the $\mathrm{I}, \mathrm{j}, \mathrm{k}$ components, $\mathrm{m} \mathrm{s}^{-1} ; u$ internal energy (physical), $\mathrm{J} ; g$ gravitational acceleration, $\mathrm{m} \mathrm{s}^{-2} ; T$ temperature, $\mathrm{K} ; \lambda$ thermal conductivity, $\mathrm{W} \mathrm{m} \mathrm{m}^{-1} \mathrm{~K}^{-1} \Gamma$ stress tensor, $\mathrm{N} \mathrm{m}^{-2}$.

The surfaces of fluid domain, which were in contact with water jacket were characterized by a specified temperature. The heat flux from the wall to the domain for laminar flow is given the temperature gradient and for turbulent is the following [4]:

$$
\mathrm{q}_{\mathrm{w}}=\mathrm{h}_{\mathrm{c}}\left(\mathrm{T}_{\mathrm{w}}-\mathrm{T}_{\mathrm{nw}}\right)
$$

where $q_{w}$ heat flux, $\mathrm{W} \mathrm{m}{ }^{-2} ; T_{w}$ specified temperature, $\mathrm{K}$; $T_{n w}$ near wall temperature, $\mathrm{K} ; h_{c}$ coefficient which involves the use of turbulent wall functions, $\mathrm{W} \mathrm{m}^{-2} \mathrm{~K}^{-1}$.

The model did not take into account the viscous dissipation. Mass flow of the operating medium of 8.5 $\mathrm{m}^{3} / \mathrm{h}$ has been assumed as the inlet boundary condition. The density of water has been determined by the inlet temperature (in line with the experimental data).

Temperature values in the selected points of the combustion chamber were used to make assumptions related with temperature of the walls. The temperature values of internal walls of the water jacket were in the range of $343-453 \mathrm{~K}$.

The morphology of material has been set as a continuous fluid. Assumed reference pressure was equal 0,4 bar. Buoyancy model was skipped. Computations have been initialised with the temperature of the domain $293,15 \mathrm{~K}$.

The $\mathrm{k}$ - epsylon $(\mathrm{k}-\varepsilon)$ model of turbulence has been used due to of its completeness, generality and lack of requirement to analyze the inflation layer at the early stage of research. Wall function has been set as a scalable. In the $\mathrm{k}-\varepsilon$ model and its extensions, the turbulent viscosity is calculated as a function of turbulence parameters: kinetic energy $\mathrm{k}$ and its dissipation rate $\varepsilon$.

The k- $\varepsilon$ model and its extensions require solving partial differential equations for turbulent kinetic energy and its dissipation rate. The so-called upwind method was implemented to solve this computational problem.
Turbulence intensity in the water domain was assumed as medium (intensity $=5 \%$ ).

The initial parameters of the fluid domain in the model were defined based on the real conditions at the beginning of the experimental studies the temperature of the operating medium in the water jacket was assumed to be approximately $293,15 \mathrm{~K}$.

Results obtained by simulations described above were processed in the CFX postprocessor and presented in the next chapter.

\section{Results of the numerical simulations and discussion}

As a first step of the results analysis, investigation of flow parameters of the operating medium in the water jacket has been performed. figure 10 presents a detailed view of a part of the water distribution system. The whole element is constructed as a duct in the bottom part of the back and side areas of the jacket. Small holes visible at the top of the main duct in the mentioned figure provide fresh, cold water from the inlet and distribute it along the walls. Vortexes are visible especially in the area between the structure supports (vertical, parallel elements). The vortexes provide the appropriate mixing of water near the hot wall of the water jacket and of the medium in the central part of the domain.

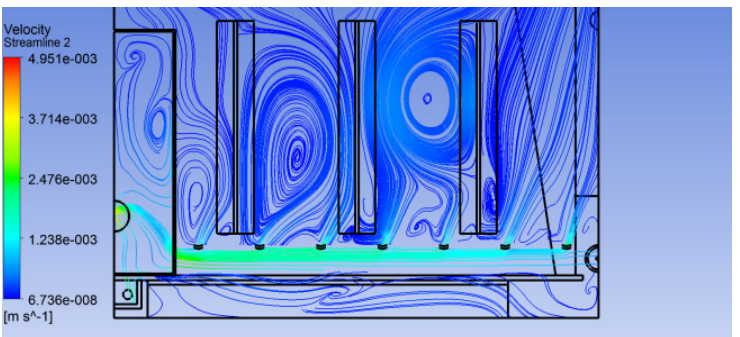

Figure 10. Characteristics of the water distribution system operation (the back wall of the water jacket)

The velocity vectors in figure 11 show the difference in the behaviour of the operating medium in the area of the back wall of the jacket and the wall separating the chambers.

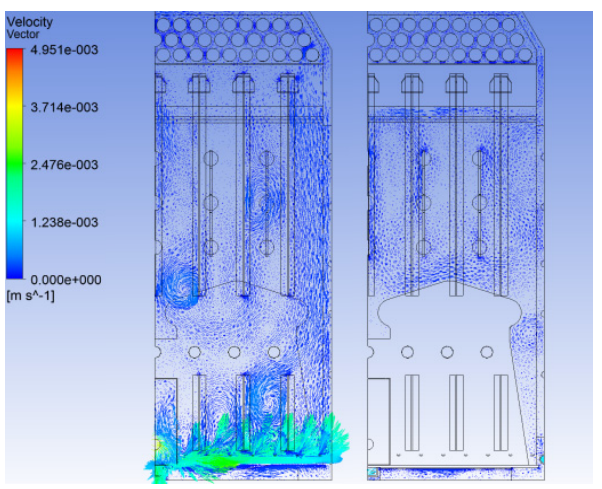

Figure 11. Rear view of cross-sectional planes of two areas of the water jacket. Starting from left: area of the back wall, separation wall between the primary and the secondary combustion chamber. The method of water distribution also affects the heat transfer efficiency within the heat exchanger. 
Due to the presence of outlets from the water distribution system and their location directly under the outlet duct, the water exchange is much higher in the first case than in the vicinity of the separation wall, where sudden changes in the flow direction (area of water jacket above the primary combustion chamber) additionally affect the flow resistance. The result is the risk of overheating of the element. In modern units such problems are solved by adding an extra pipe connected to the water distribution system in the problematic part of the structure.

Figure 12 shows vector velocity distribution on three planes perpendicular to the exchanger pipes. In the first and second example the flow of medium between the pipes in the central part of the exchanger is limited by thickness of the gap between the element and the wall of the water jacket. The effect of the increased flow, which is visible in all three cases is the most intense in figure "a" showing the flow near the front of the boiler (front part of the heat exchanger, the exhaust inlet). In this region, the heating medium transported from the bottom of the water jacket turns back to reach the outlet pipe

a)

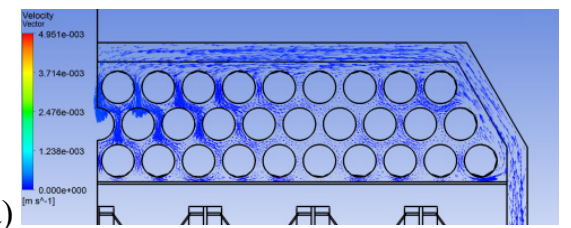

b)

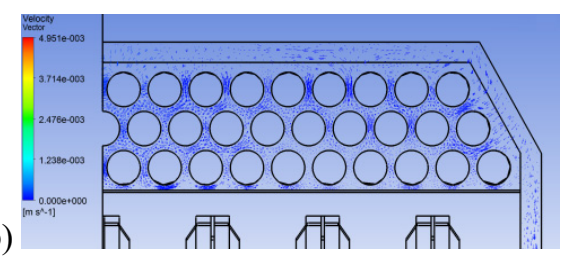

c)

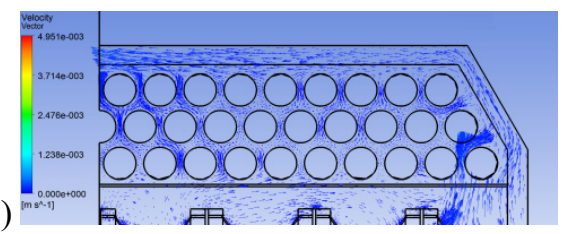

Figure 12. Cross-sectional planes of three areas of the heat exchanger: a) near the front of the boiler (exhaust inlet), b) in the central part of the heat exchanger, c) near the back side of boiler (exhaust outlet).

The way of water distribution also affects the heat transfer efficiency within the heat exchanger. Figure 12 shows the vector velocity distribution on three planes perpendicular to exchanger pipes. In the first and second example the flow of medium between the pipes in central part of the exchanger is limited by the thickness of the gap between the element and the wall of the water jacket. The effect of the increased flow, which is visible in all three cases is the most intense in figure "a", displaying the flow near the front of the boiler (front part of the heat exchanger, the exhaust inlet). In this region, the heating medium transported from the bottom of the water jacket turns back to reach the outlet pipe. Example "c" is unique because of the direct flow of water from the distribution system. The area of the heat exchanger is penetrated.

Figure 13 presents a general view of the temperature distribution. The figure illustrates the boundary conditions based on experimental data obtained in 10 minutes of the first measurement (chapter 4). Regions characterised by the highest temperatures are in contact with the secondary combustion chamber where the process of combustion occurs in the first stage of the process.

It is evident that the temperature level of the medium is lower in the area adjacent to the separation wall (between the chambers).

The heterogeneity of temperature distribution is related mostly to the position of the cold water inlet and presence of many structural elements inside the water jacket. Figure 14 presents the variation of the water temperature along the exhaust pipe in the heat exchanger. Due to the exhaust flow direction, the medium is warming up more efficiently in the front part of the exchanger.

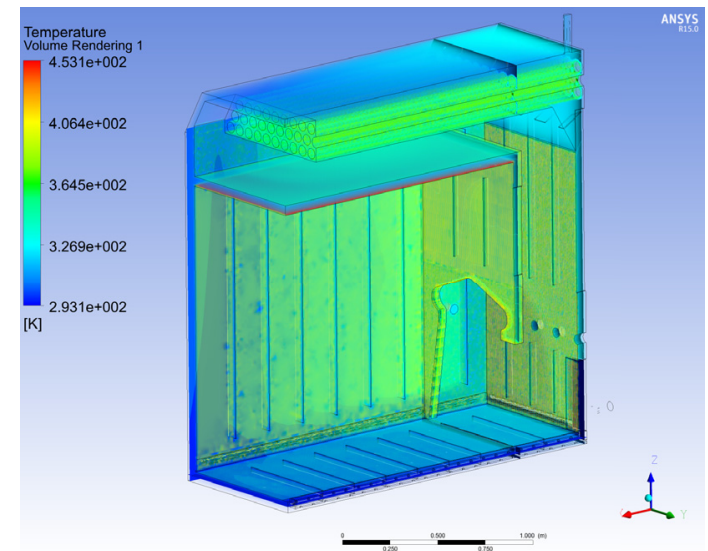

Figure 13. General view of the temperature distribution in the operating medium of the RM40 boiler.

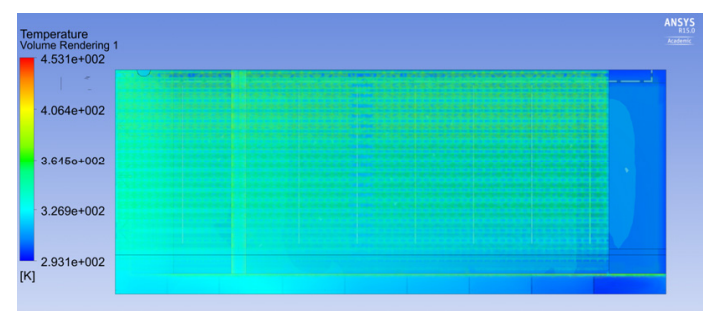

Figure 14. Top view of the heat exchanger.

To allow more accurate studies of the heating process inside the medium, the model has been supplemented with so-called monitor points. 


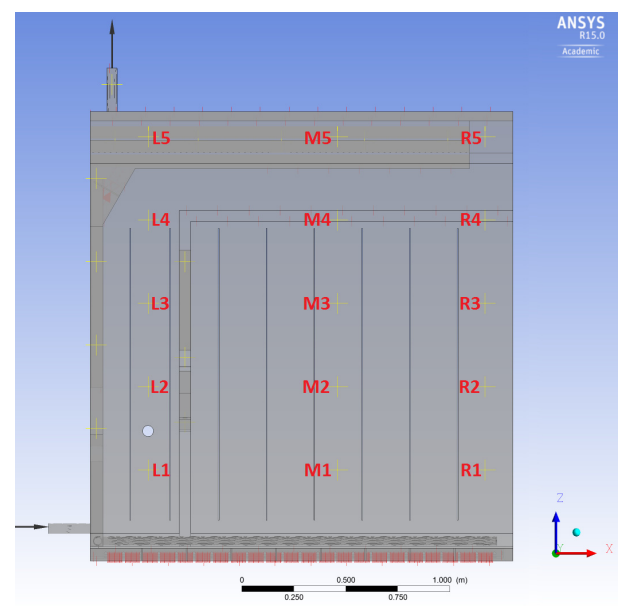

Fig. 15. The monitor points chosen for the controll of the temperature inside the region of the side wall of water jacket.

Coordinates of the specified points were selected to observe variation of the temperature of flowing operating medium. The points have been groupped in the vertical rows, what allows analysing the similarity of the thermal phenomenons in chosen areas of the domain.

Numbers assigned to the given points are consistent with the general direction of the operating medium flow (from the bottom, up to the roof). Varations of the temperature along the rows are shown in figure 16. Much lower temperature for points placed near to the front of the boiler are related with the impact of the relatively cold water flowing from the floor part of the water jacket. Due to analogous construction of the side wall and the floor part (transverse supports), behaviour of the medium in mentioned areas is similar flux of water is partially directed to the front of the boiler (resulting that the front bottom corner is relatively cold).

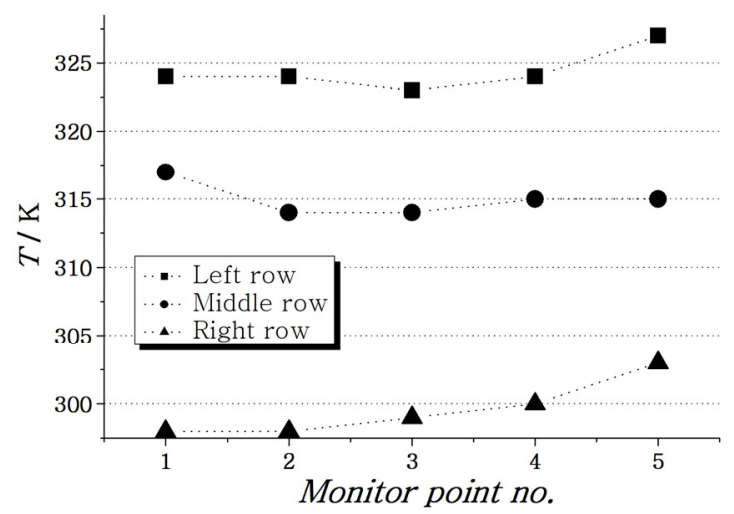

Fig. 16. Values of the temperature sensed in the selected points of the side wall of the water jacket.

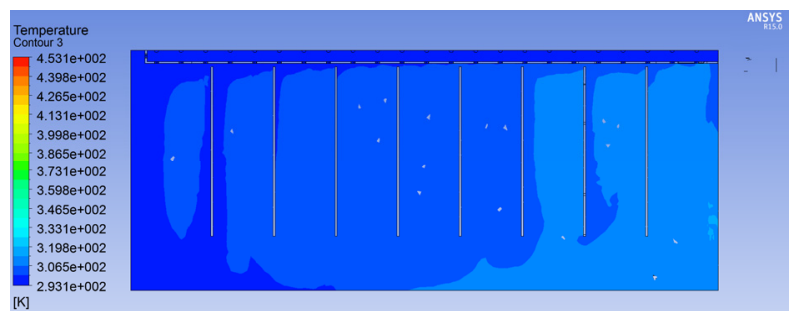

Fig. 17. Distribution of temperature in the area of the water jacket floor.
Desrcibed phenomenon is confirmed by figure 17 , which presents distribution of temperature in the floor region of the domain. The hotest part of this area is still relatively cold in comparison with the central and rear part of the side wall.

The important fact is that points marked by " 5 " in the figure 16 are situated in the area of the heat exchanger, outside the vertical part of the wall. Except the second case (central row of the monitor points), temperature in this part of the analysed area is the highest. The second analysed part was the heat exchanger. The selected monitor points are partially visible in figure 18 . Rows marked by "L" and "M" are parallel to the "C" row. All points are located centrally between pipes of the heat exchanger.

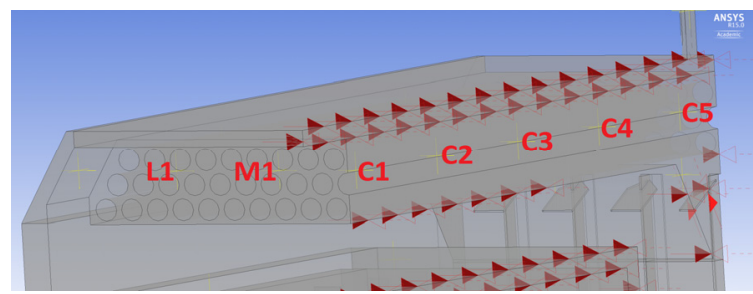

Fig. 18. The monitor points chosen for the controll of the temperature inside the region of the heat exchanger.

The temperature distribution along the analysed area is analogous for all curves. Almost the same values are observed on the beginning and the end of the middle and the central row. Characteristic of heating of the medium presented in figure 19 is in relation with the vectors distribution, showed in figure 12 .

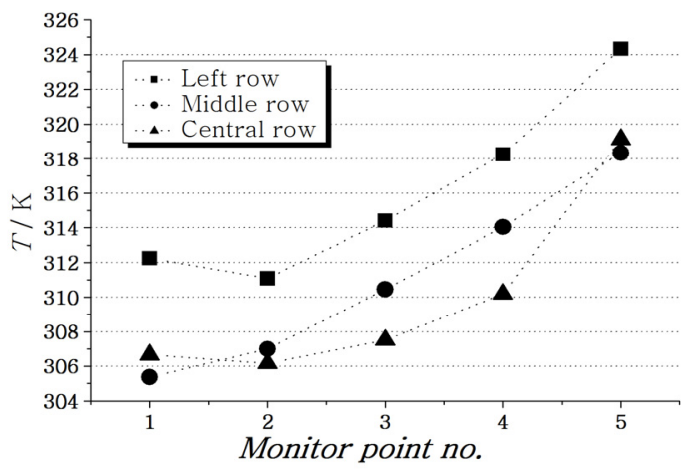

Fig. 19. Values of the temperature sensed in the chosen points of the heat exchanger.

Shape of the heat exchanger results in the directed flow of medium, what is disturbed only in part near to the connection with the vertical wall of the boiler. Mentioned phenomenon influences on the temperature of water in considered region. 


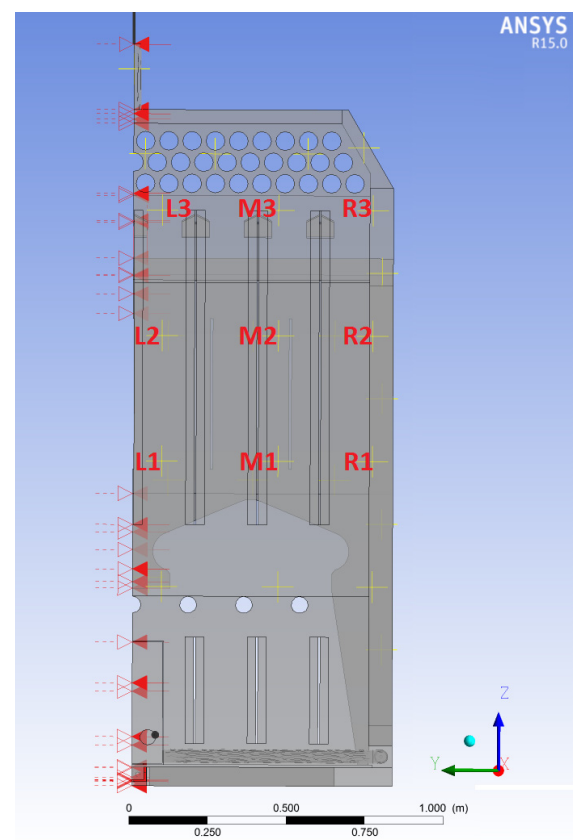

Fig. 20. Monitor points chosen for the control of the temperature inside the region of the rear wall of water jacket.

Third interesting part of the water jacket construction is rear wall, equipped with series of supports and additional barriers resulting from the installation of air supply system (visible for example in figure 11), which is presented with the marked monitor points in figure 20 .

Such fact makes temperature distribution in this area the most complicated. Various velocity of water between welded metal elements result in different temperature in relatively nearby areas.

Unexpected effect of cooling in case of the left and middle row of points results from the mixing with operating medium from different parts of the water jacket (number of connections).

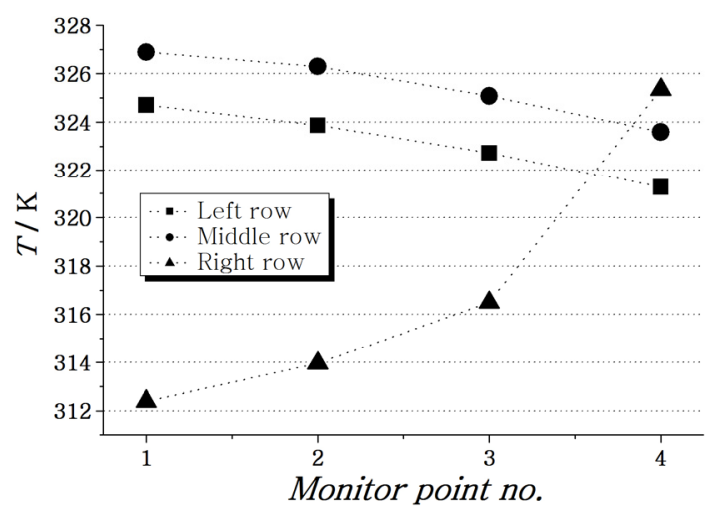

Fig. 21. Values of the temperature sensed in the chosen points of the rear wall of the water jacket.

Different shape of the curve created using values taken from right row of points is related with near vicinity of the medium from side part of the water jacket. Results obserwed for the lowest and the highest points of the "R" set, in this case are consistent with the data presented on the charts in figures 6 and 7, created using experimental data.

The highest values of temperature were observed in the wall separating combustion chambers, where value for the most exposed on high temperature part has reached about $340 \mathrm{~K}$. The mentioned area was identified as the region strongly endangered by overheating, what is in agreement with distribution of the fluid velocity vectors (figure 11).

\section{Conclusions}

The paper presents the possibility of using numerical computation tools to expand the range of available data related to the operation parameters of biomass-fired heating units. CFD tools allow gaining valuable information related to the operation of the studied device. As a result of the investigations, data on the temperature distribution inside the water jacket, as well as details of the operating medium flow has been obtained. Practical issue related with the exploitation and optimization of the boiler design has been developed. It is recommended to increase the water distribution in the area of the wall separating the primary and the secondary combustion chambers, to avoid overheating of this region by increasing the water flow velocity. As a next stage of the study, the impact of the mentioned design changes and various working conditions on the efficiency of the device operation will be examined.

This study was carried out under contract (11.11.210.217) AGH-University of Science and Technology, Faculty of Fuels and Energy, Cracow, Poland.

\section{References}

1. T. B. Johansson, H. Kelly, A. K. N. Reddy, R. H. Williams, Renewable energy, Sources for fuels and electricity (1993).

2. B. Sørensen, Renewable Energy. Its physics, engineering, environmental impacts, economics \& planning (2000).

3. W. Kordylewski, Spalanie i Paliwa (2008) - in Polish. Available online at: http://www.metalerg.pl (access : 15.09.2014).

4. ANSYS WORKBENCH 15 help files 